\title{
Perioperative Management of Anticoagulants and Antiplatelets
}

\author{
Dr. Haydi Sayed, MD \\ Lecturer of internal medicine, \\ Department of hematology and bone marrow transplantation, \\ Ain Shams University
}

\section{Capsule summary adopted from the American College of Chest Physicians Evidence-Based Clinical Practice Guidelines}

1 Evaluate the thromboembolic risk and hemorrhagic risk of the individual patients

2 Consider temporary cessation of the drug in procedures that carry a significant risk of bleeding

3 Low thromboembolism and bleeding risk

Warfarin may be continued with relatively low INR 1.5-1.8 for minor procedures

4 For high bleeding risk with low-thromboembolism-risk group

Warfarin can be withheld for 5 days before surgery without any bridging anticoagulation with unfractionated or low molecular weight heparin

5 High-thromboembolism-risk patients

Generally such patients should be considered for a more aggressive perioperative management strategy with bridging therapy

6 As compared with warfarin, patients on NOACs are less likely to require bridging therapy due to their short half-life

\section{ANTIPLATELET AGENTS}

1 Use of DAPT following percutaneous coronary procedures and following acute coronary syndrome are common

2 Current recommendations for DAPT range from 4 weeks in patients undergoing elective stenting with bare metal stents to up to 12 months in patients with drug-eluting stents or patients undergoing coronary stenting for acute coronary syndrome

3 Low-dose aspirin alone does not substantially increase the risk of clinically important bleeding after invasive procedures and can usually be continued during surgery

4 If a patient is to undergo high-bleeding-risk surgery and an antiplatelet effect is not desired, clopidogrel, prasugrel and ticagrelor should be discontinued 5-7 days prior to the procedure

5 Early, effective communication between GPs and specialists is useful in managing high-risk patients on anticoagulant/antiplatelet agents during the perioperative periods

DAPT, dual antiplatelet therapy; NOACs, new oral anticoagulants 
Figure 1.Peri-Operative Management of Warfarin-Treated Patients Before and After Surgery/Procedure

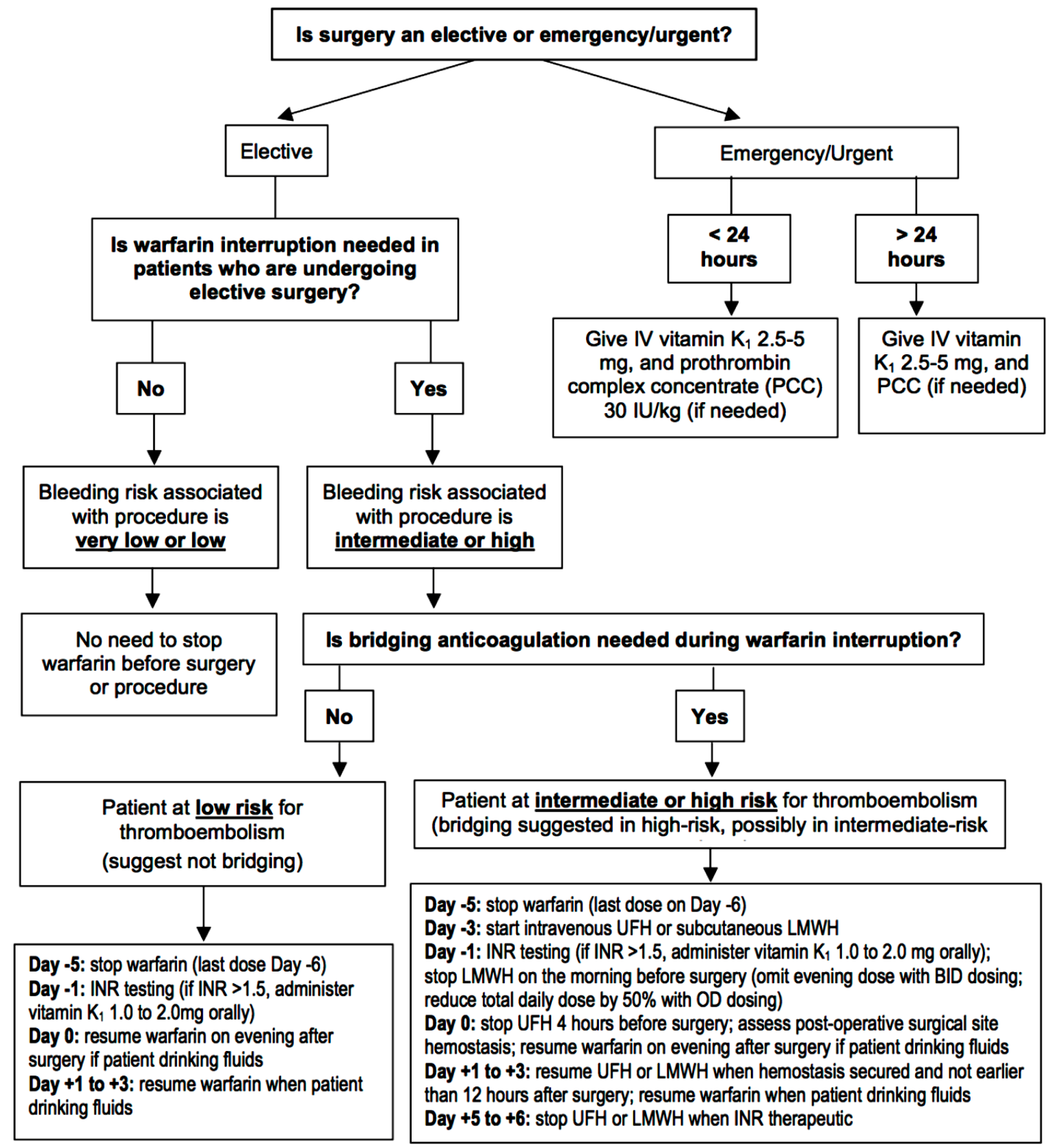




\section{Post-Operative Resumption of Bridging Anticoagulation}

- High-Risk Bleeding Procedure:

O Therapeutic-dose LMWH/UFH, starting 48-72 hours after surgery

- Alternate management: low-dose LMWH, starting 12-24 hours after surgery (i.e. day after surgery) or resume warfarin alone with no post-operative $\mathrm{LMWH} / \mathrm{UFH}$

- Moderate-Risk Bleeding Procedure:

O Therapeutic-dose LMWH/UFH, starting 24-48 hours after surgery

- Low-Risk Bleeding Procedure:

- Therapeutic-dose LMWH/UFH, starting 12-24 hours after surgery (i.e. day after surgery).

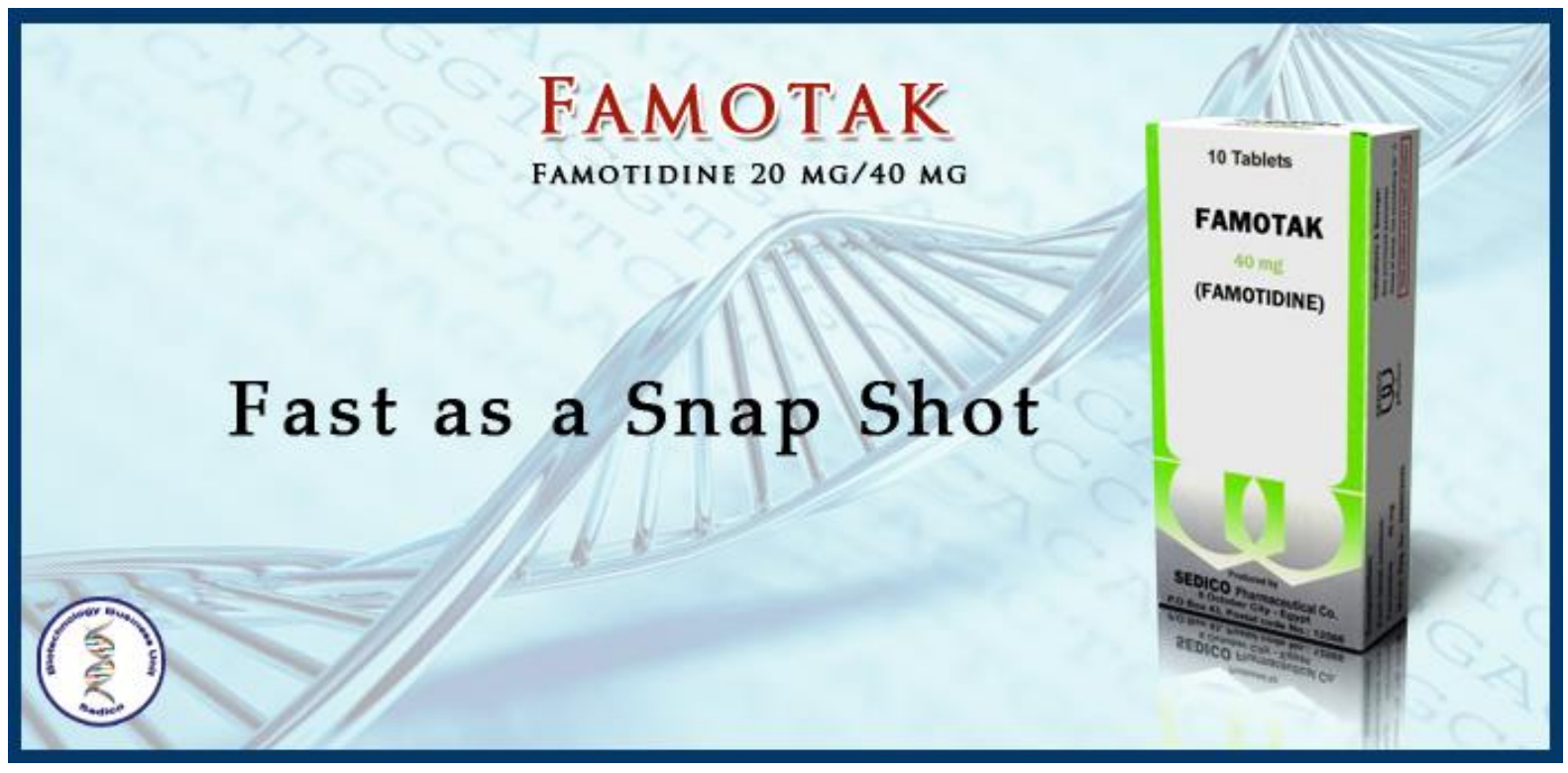

